\title{
A classification model on tumor cancer disease based mutual information and firefly algorithm
}

\author{
Saadya Fahad Jabbar ${ }^{1}$ \\ ${ }^{1}$ College of Education for Human Science - Ibn Rushed, Universit of Baghdad, Baghdad, Iraq
}

\section{Article Info}

Received May 33, 2019

\section{Keyword:}

Feature Selection

Firefly Algorithm

Cancer Disease

Mutual Information

Classification

\begin{abstract}
Cancer is a globally recognized cause of death. A proper cancer analysis demands the classification of several types of tumor. Investigations into microarray gene expressions seem to be a successful platform for revising genetic diseases. Although the standard machine learning (ML) approaches have been efficient in the realization of significant genes and in the classification of new types of cancer cases, their medical and logical application has faced several drawbacks such as DNA microarray data analysis limitation, which includes an incredible number of features and the relatively small size of an instance. To achieve a reasonable and efficient DNA microarray dataset information, there is a need to extend the level of interpretability and forecast approach while maintaining a great level of precision. In this work, a novel way of cancer classification based on based gene expression profiles is presented. This method is a combination of both Firefly algorithm and Mutual Information Method. First, the features are used to select the features before using the Firefly algorithm for feature reduction. Finally, the Support Vector Machine is used to classify cancer into types. The performance of the proposed system was evaluated by using it to classify datasets from colon cancer; the results of the evaluation were compared with some recent approaches.
\end{abstract}

\section{Corresponding Author:}

First Author,

College of Education for Human Science - Ibn Rushed,

Universit of Baghdad,

Baghdad, Iraq,

Email: saadya.fahad@ircoedu.uobaghdad.edu.iq

\section{Introduction}

Cancer is a term that refers to diseases that result from an uncontrolled body cell division. Such uncontrolled cell division results in the development of lumps known as a tumor. These abnormal cells invade the immune system and spread through the blood vessels and lymph system to other parts of the body. There are more than 100 types of tumors which are mainly named based on where they are formed in the body or based on the type of tissue they develop from. Cancers can be classified into six major classes based on the type of tissue involved: Carcinoma, Leukemia, Sarcoma, Myeloma, Lymphoma, and Mixed Types. based on the initial place of origin, cancers can be specified as breast, lung, prostate, liver, kidney, oral, or brain cancers[1], [2].

A proper identification of cancer is necessary for its medical diagnosis; it is also necessary for an effective treatment and minimization of toxicity on patients. Traditionally, cancer identification and classification have been through the identification of the morphological features of tumorous tissues. However, there are several limitations of these conventional methods and as such, there is a need to develop methodologies which can effectively differentiate between cancers subtypes. DNA microarrays have made it possible to simultaneously 
observe the level of expression of several genes [3] and have facilitated the ascent of computational analysis, including ML techniques.

These techniques are useful for pattern extraction and classification model development from the gene expression database; they have also been helpful in cancers prognosis and management[4]. DNA microarray technologies have found application in the prediction of cancer diseases and have served as an effective platform for gene expression analysis in several experimental studies. With microarray technologies, the level of expression of several genes can be analyzed from 2 test cells. regarding how to get the samples cells, there is a need for essential investigations such as accurate diagnosis, disease improvement, response to medication, and post-treatment prognosis are necessary[5]-[7].

Several feature selection frameworks have been developed over time and their review may be necessary to understand their successes and limitations [6]. Several studies have previously been involved in the study of the effectiveness of an attribute subset in deciding an optimal. Essentially, feature selection is an optimization problem; however, a well-organized process of discriminative gene selection from microarray gene expression data for cancers, diagnosis has been recommended. Further, the need for dimension reduction prior to classification with gene expression microarray approach has been outlined. Recently the swarm-based methods and evolutionary methods like Ant Colony Optimization (ACO)[8]-[10], Genetic Algorithm (GA) [11]-[13], Artificial Bee Colony (ABC)[14], [15] Particle Swarm Optimization (PSO)[16], [17] and Harmony Search Algorithm (HSA) have been used to handle the problems of features selection[18], [19]. Firefly algorithm (FA) was developed by Yang [20], [21] as a swarm-based metaheuristic. FA has attracted much research interest owing to its efficiency in handling optimization issues [22]-[25].

In this paper, the issue of human cancer disease classification was tackled using gene expression profiles. This study presents a new approach for the analysis of microarray datasets and for an efficient classification of cancer[26], [27]. In the new approach, Mutual Information (MI) is used as a feature selection model for the selection of the most relevant features, while Firefly algorithm (FA) was deployed for feature reduction. Support Vector Machine (SVM) was used as the classification model for the evaluation of each solution achieved by the Firefly algorithm. The remaining part of this paper is arranged in the following way: section 2 provides an overview of FA and MI, while section 3 explained the proposed classification algorithm in this study. In section 4, the results of the study are presented, while the conclusions drawn from the study are provided in section 5 .

\section{Overview}

Optimization means determine the current best solution to the problems which can not be solved in a deterministic polynomial time, or NP hard problems [28]-[31]. In most real-world optimization tasks, finding optima requires an expensive computation process. Some of the study limitations such as computation resource constraints and project time requirements have necessitated the need to make the optimization process less complicated and rapid[32], [33]. Most of the standard optimization frameworks require several function evaluations and usually produce satisfactory results due to their inherent special information transfer mechanism of using several first-choice solutions in a range of fitness evaluations. The need to evaluate each candidate resolution makes these processes to demand much computing resources and execution time. Consequently, efforts have been devoted to the development of efficient optimization algorithms for the evaluation of several functions. Several novel approaches have been proposed in recent times with some satisfactory performances with fewer function evaluations [28], [34]-[38].

Feature selection is a pre-processing method of selecting the most informative genes which can distinguish groups (cancer subtypes in this case). The major aim of the feature section is to establish a reduced set of features from a dataset in a bid to reduce the initial dimensionality of the feature space. In general, studies on cancer classification require the deployment of formal feature selection strategies for the purpose of lowering the computational demands in experimental responsibilities which helps to evaluate and explore data in such domain, as well as to provide a programmable solution in terms of structure and the number of features[39][41]. 


\subsection{Mutual Information}

In the filter based methods, variables are ranked without any form of dependence on the classifier, and some of such performance measures are Fisher score, Pearson correlation coefficient, and Information theory-based measures [42] [43]. Such techniques are advantageous because they are easy to implement, computationally less expensive, and provides a more generalizable feature subset since they are not dependent on any classifier [44]. Having said that, their major problem is that they cannot exploit specific ML algorithm characteristics which are intended for use, and as such, rarely achieve the highest level of classification accuracies.

MI refers to the specific information shared by 2 variables. Through entropy, the conveyable information from a variable can be quantified but the major point of interest is the level of variables overlaps in the recorded variables. This is important when considering the effectiveness of one variable in the prediction of the other; a higher level of shared information implies that a similar information source is being measured:

$$
I(X: Y)=H(X)-H(X \mid Y)=H(X)+H(Y)-H(X, Y)
$$

Where $I$ represents the value of weight of individual feature, while $H$ denotes the entropy value. Entropy is calculated by the summation of all the probability distribution of values of the feature, multiplied by the natural $\log$ of those probability distribution, as follows:

$$
H(X)=-\sum p(x) \log p(x)
$$

Where $\boldsymbol{x}$ represent a value of the set $\boldsymbol{X}$, while $\boldsymbol{p}(\boldsymbol{x})$ represents the probability distribution of $\boldsymbol{x}$.

\subsection{Firegly Algorithm}

The FA is a recently developed nature-inspired metaheuristic which was inspired by the light flashing pattern of fireflies [20], [45] There are three general rules guiding the fireflies in the FA:

- The fireflies are randomly attracted to each other (unisex).

- The level of attractiveness of a firefly depends on its brightness; attractiveness relates inversely with the distance between any 2 fireflies.

- The brightness of a firefly is directly related to the value of its objective function.

There are two important issues in the FA, and these involve the changes in the light intensity and the attractiveness formulation. The attractiveness of a firefly simply depends on its brightness, but being that attractiveness decreases with distance between 2 fireflies, it appears that weaker intensities imply less attractiveness. Thus, both light intensity variation and attractiveness ought to be monotonically decreasing function often formulated thus:

$$
E(r)=E_{0} e^{-\gamma r^{2}}
$$

where $r=$ inter-firefly distance, $E_{0}=$ initial light intensity, and $\gamma=$ coefficient of light absorption which controls light intensity variation (the value of $\gamma$ is usually fixed in the FA). The attractiveness of a firefly in the FA is directly related to the light intensity as defined in Eq. 2.26.

$$
\beta(r)=\beta_{0} e^{-\gamma r^{2}}
$$

where $\beta_{0}=$ attractiveness at $r=0$. Then, the attraction of the $i$ th firefly to the $j$ th firefly can be formulated thus:

$$
x_{i}=x_{i}+\beta\left(x_{i}-x_{i}\right)+\alpha\left(\operatorname{rand}-\frac{1}{n}\right)
$$


where $a=$ randomization parameter, $r a n d=$ a uniformly distributed random number generator in the range $[0,1] . r_{i, j}=$ distance between the $i$ th and $j$ th firefly which can be formulated thus:

$$
r_{i, j}=\sqrt{\sum_{d=1}^{D}\left(x_{i, j}-x_{i, d}\right)^{2}}
$$

where $D$ is the size of an optimization problem. The pseudocode of FA is given in the figure follow.

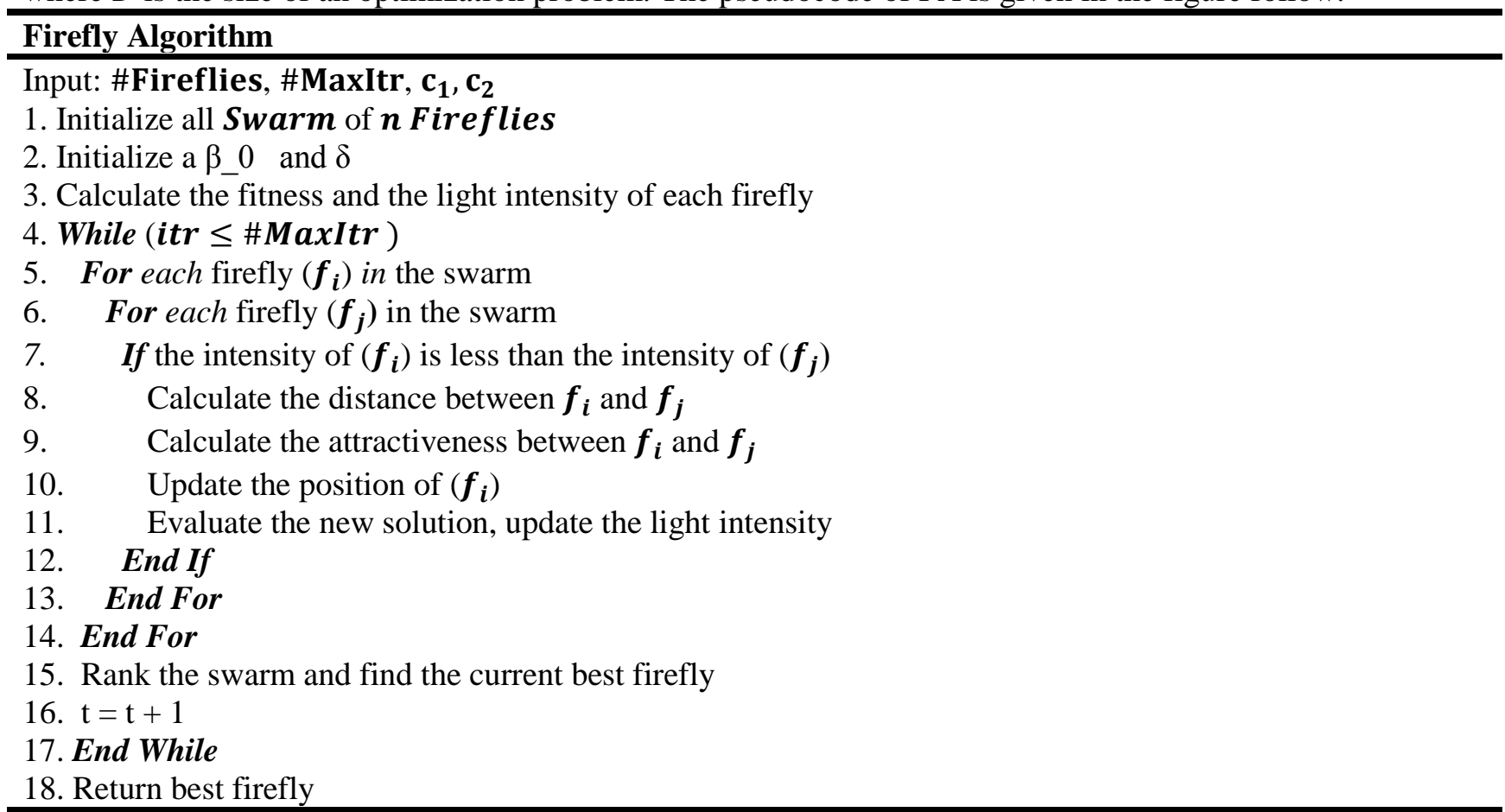

Figure1. The pseudocode of FA

\section{The Proposed Algorithm}

Feature selection algorithms are mainly developed due to the need for finding better subset features that will offer better performance accuracies. The proposed model is divided into two main stages. In the first stage, the features in the dataset are filtered by using MI. The selection process depends on the high weights of the features. Then, the second stage is the wrapper feature selection, which is represented by the firefly algorithm, which selects the most relevant features from the results of the first stage. Figure 1 portrays the general structure of the wrapper feature selection method, while Figure 2 displays the proposed model.

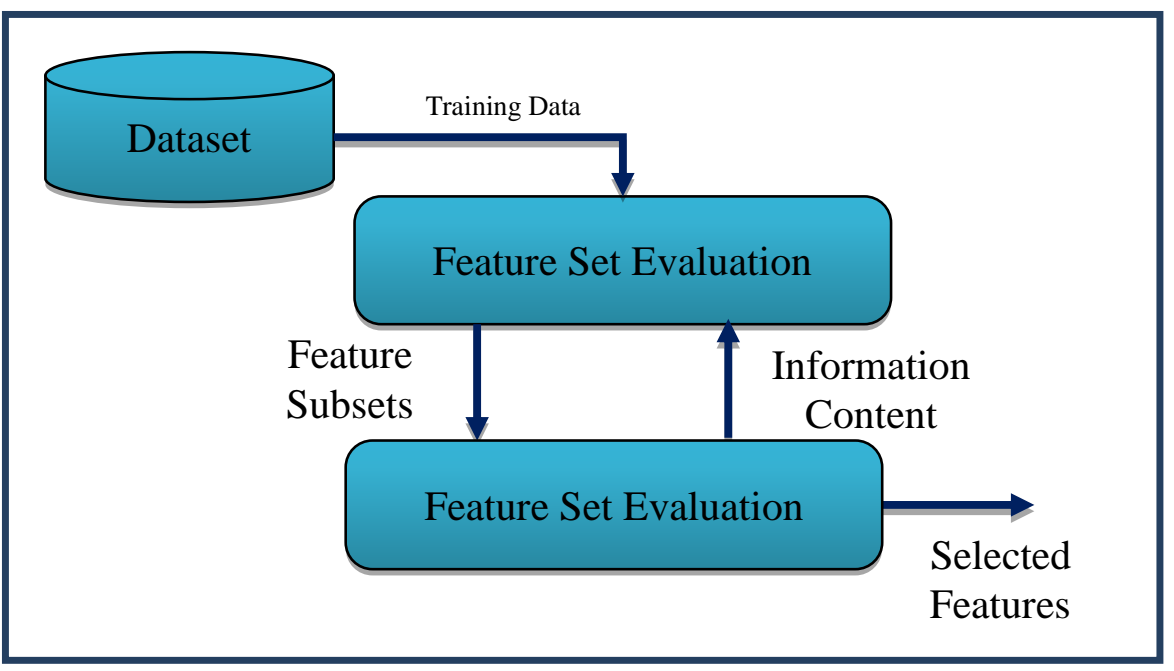

Figure 2. Wrapper Feature Selection Method 
In the second stage, all the Fireflies in the swarm are initialized in a binary sequence, unlike in the traditional wrapper model where the Fireflies are initialized with randomly selected features. The proposed BFA is comprised of four (4) major steps - initialization, fitness function, attractiveness calculation, and positional updating. These steps are further explained in the following subsections.

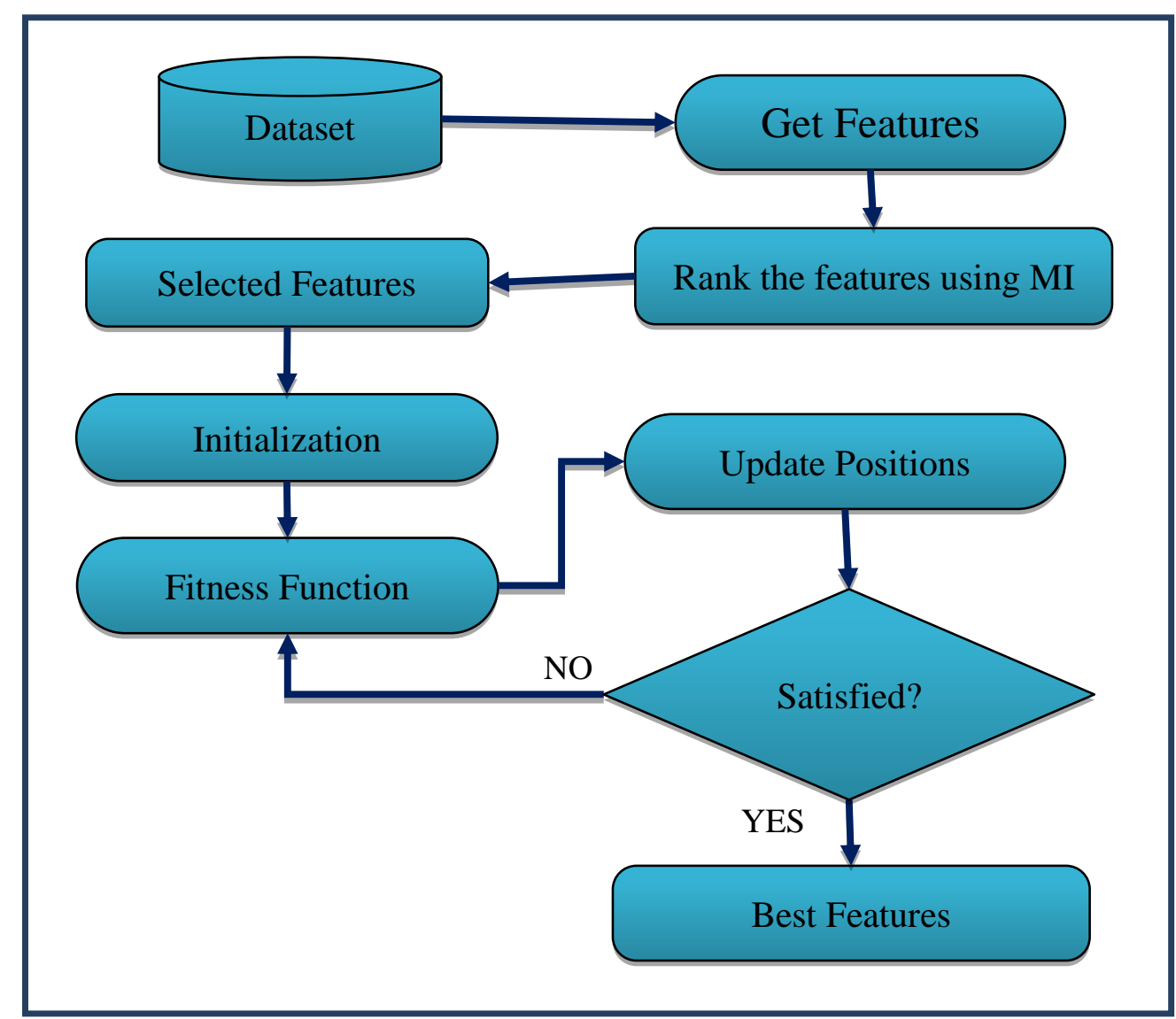

Figure 3. The proposed Model

\section{A. $\underline{\text { BFA Initialization }}$}

All the Fireflies in the search space is initiated in this step using a random number in the range of [0,1]. The position of each firefly in the search space is represented by these random numbers. The position of each firefly is calculated using equation 1 ,

$x=[u-l] x$ Rand $[0,1]+l$

Where $u=$ upper bound [1.0] and $l=$ lower bound [0.0]

The generated sequence is further converted into a binary sequence using the relation in equation 2: -

$b_{i}=\left\{\begin{array}{l}1, \text { sigmoid }(x)>u[0,1] \\ 0, \text { otherwise }\end{array}\right.$

Where $x_{i}=$ position of each firefly, sigmoid $\left(x_{i}\right)=1 /\left[1+e^{-x_{i}}\right], u=$ uniform distribution. $b_{i}=$ binary sequence, $1=$ chances that a feature will be selected, and $0=$ chances that a feature will not be selected. Each initiated Firefly in the swarm has its own position based on the generated number of each Firefly.

\section{B. Calculation of the fitness function $(F F)$}


In the proposed algorithm, the FF is determined to minimize the rate of classification error over the validation set of training data, as shown in equation 2, while maximizing the number of irrelevant or non-selected features. The FF of the algorithm was calculated using a classifier. Here, the Support Vector Machine (SVM) was applied to determine the classification accuracy.

$$
\text { Error }=100-A
$$

where $\mathrm{A}=$ accuracy rate of the classifier, in other words, the 5-fold cross-validation error rate after training the SVM. Equation 4 is used to calculate the intensity of each Firefly based on the error value.

$$
I\left(F_{i}\right)=1 /\left(1+\text { Error }^{2}\right)
$$

Calculation of the attractiveness of each firefly. To calculate the level of attractiveness $\beta$ of each Firefly, equation 5 was deployed:

$$
\beta(r)=\beta_{0} \times e^{-\gamma r^{2}}
$$

where $r=$ the distance between 2 fireflies (calculated using equation 6), $\beta \_0=$ the attractiveness of a firefly at the initial case $(\mathrm{r}=0)$.

$$
r_{i j}=\left|x_{i}-x_{j}\right|
$$

where $\mathrm{X}=$ real positional values of each firefly earlier calculated using the information gain ratio equation.

This distance between 2 fireflies is calculated using the hamming distance method, where each bit of Firefly $i$ is subtracted from Firefly $j$. In this method, the distance is represented by the difference between the binary strings of the two Fireflies. This method improves the ability of the FA to work better with binary features than working with continuous values.

\section{Updating the position of the best firefly}

In the swarm, each firefly is attracted to a brighter Firefly. In the algorithm, the position of the brighter Firefly is updated using equation 7.

$$
x_{i}=x_{i}+\beta x\left(x_{j}-x_{i}\right)+\alpha \times(\text { Rand }-0.5)
$$

where $x_{i}$ in the first part of the relation $=$ current position of the best Firefly, while the second part of the relation expresses the attractiveness between position $F_{i}$ and $F_{j}$. The third part of the relation expresses the randomization with $\alpha$, where $\alpha \in[0,1]$.

This randomness is decremented by another constant rate $\delta$, where $\delta \in[0.95,0.97]$ such that at the final optimization stage, the value of $\alpha$ will be maximized, as in equation (8).

$$
\alpha=\alpha \times \delta
$$

\section{Results and Discussion}

The performance of the proposed methodology on colon cancer dataset was evaluated in this section. Two important criteria were considered for the observational evaluation of the performance evaluation and these are the number of genes selected and the predictive accuracy on the selected gene.

\subsection{Experimental Settings}

Dataset downloaded from the Kent Ridge Bio-Medical Dataset website (http://datam.i2r.astar.edu.sg/dataset/krbd/) was used in this study. This dataset is characterized as $\mathrm{N} * \mathrm{M}$ matrix, with $\mathrm{N}$ and $\mathrm{M}$ being the number of experimental samples and the number of genes involved, respectively. In the matrix, each cell represents the level of expression of a particular gene in a particular experiment, with the total number of samples being 62 and the number of genes being 2000. There are 22 positive $(\mathrm{P})$ and 40 negative $(\mathrm{N})$ samples in the dataset. The measurement of the results was based on the following diagnostic performance measures: Positive instances (P) and negative instances (N), True Positive (TP): the number of correctly diagnosed 
positive instances, True Negative (TN): the number of negative instances identified as positive (Type 1 error), False Negative (FN): the number of positive instances wrongly identified as negative (Type II error).

First, these performance measures were computed before being used to compute the algorithms' classification accuracy (CA) as follows:

\subsection{MIFA Results}

$$
C A=\frac{T P+T N}{T P+F N+T N+F P}
$$

In this paper, the proposed algorithm is compared to the other related works. Table 1 presents the comparison stage.

Table 1: Comparison Results

\begin{tabular}{cclc}
\hline $\mathbf{N}$ & Ref & & Method \\
\hline $\mathbf{1 .}$ & {$[46]$} & t. statistics + DT & 85.00 \\
\hline $\mathbf{2 .}$ & {$[47]$} & GA + SVM & 90.3226 \\
\hline $\mathbf{3 .}$ & {$[47]$} & GA + SVM & 85.4839 \\
\hline $\mathbf{4 .}$ & {$[48]$} & IG + + SVM & 71.0 \\
\hline $\mathbf{5 .}$ & {$[49]$} & MI + RBF SVM & 77.42 \\
\hline $\mathbf{6 .}$ & {$[49]$} & IG + DT & 77.26 \\
\hline $\mathbf{7 .}$ & {$[49]$} & GS + DT & 69.35 \\
\hline $\mathbf{8 .}$ & {$[50]$} & IG + SVM & 90.33 \\
\hline $\mathbf{1 0 .}$ & {$[51]$} & GA + ANN & 94.92 \\
\hline
\end{tabular}

Table 1 showed that the proposed hybrid algorithm attained the highest classification accuracy than the other methods. The proposed algorithm (MIFA-SVM) has selected a small number of selected features, which is 41 features. Which proved the ability of our algorithm to solve the gene selection problem.

The boxplot for the comparing the accuracy of the standard firefly algorithm with the proposed hybrid algorithm (MIFA) is given in Figure 4. It can be seen that the MIFA is more stable than the FA during all experiments, which means that MIFA selected the most relevant features from the dataset more than the firefly algorithm.

In addition to the boxplot, the performance of MIFA is compared with the original FA in terms of ROC and Recall-Precision curves in Figure 5 and Figure 6 respectively. It can be seen that the Recall-Precision curve of MIFA is consistently higher than that of FA.

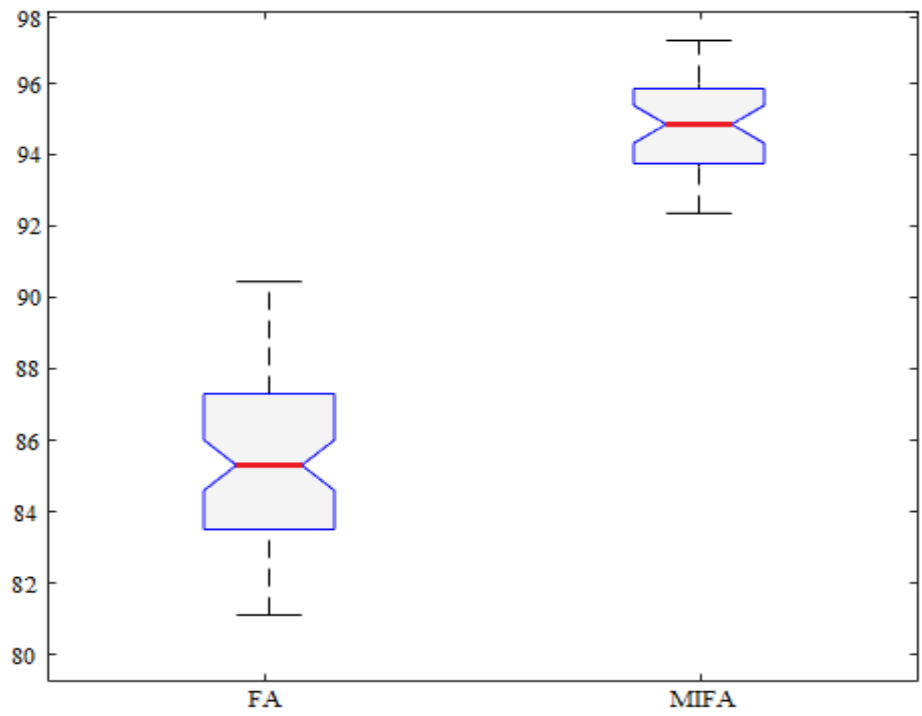

Figure 4. The box plot of MIFA and FA 


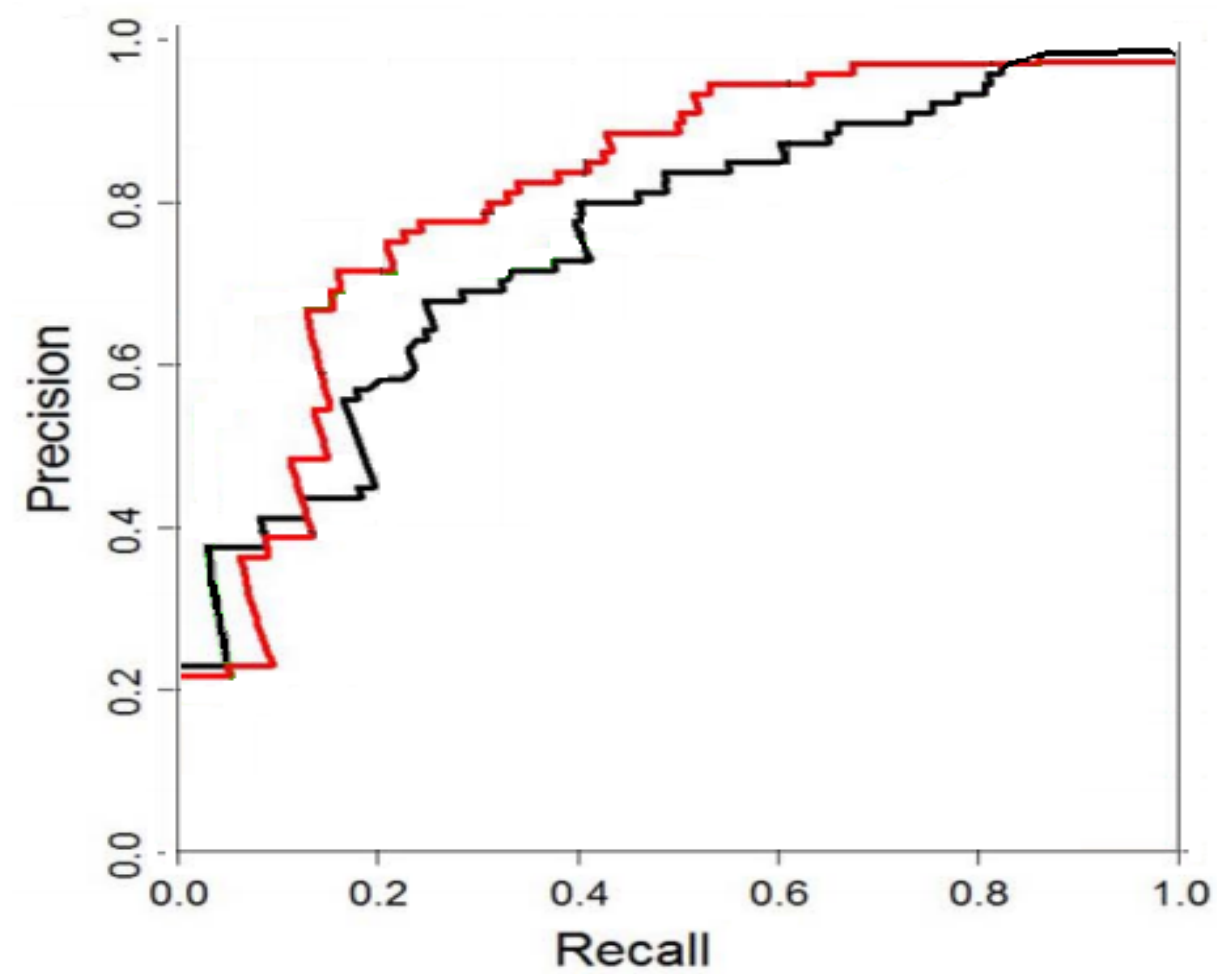

Figure 5. Precision-Recall Curve

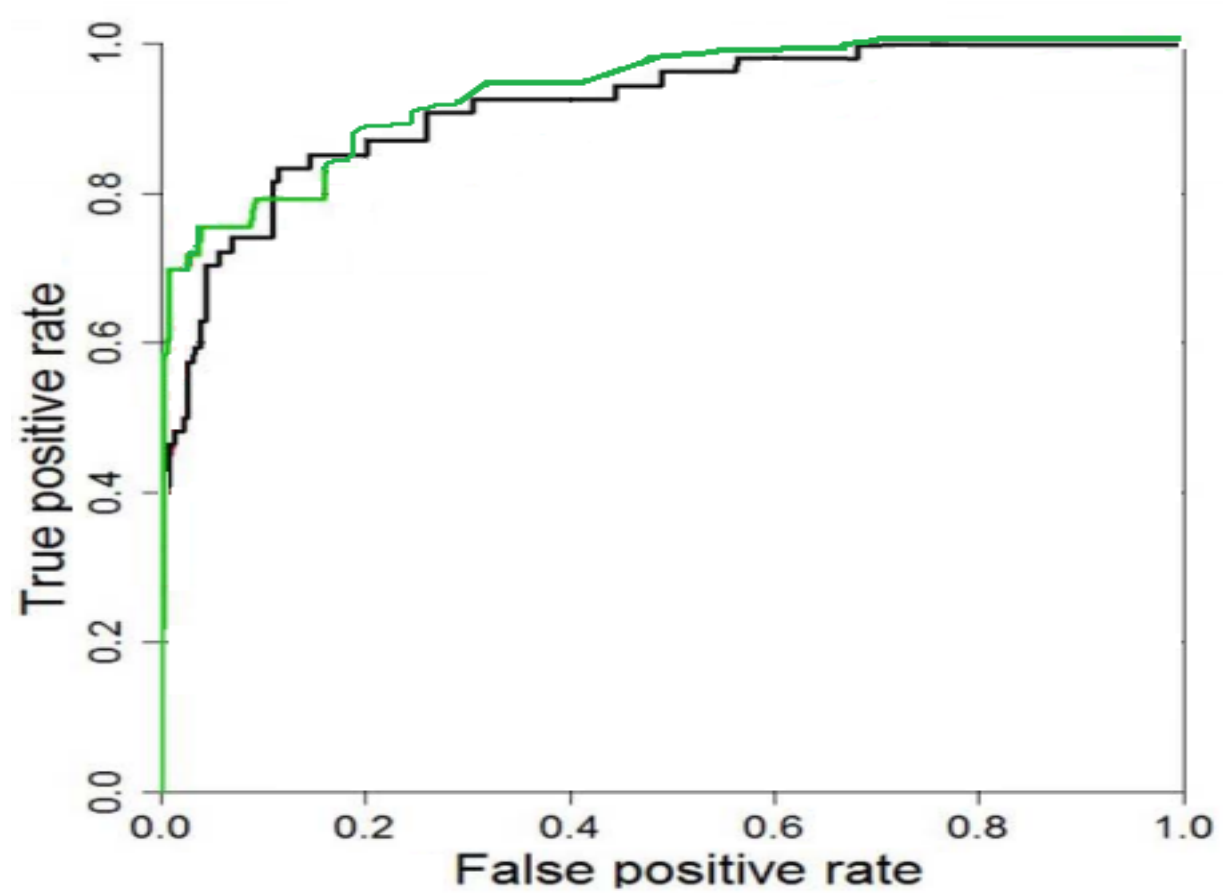

Figure 6. The Roc Curve

\section{Conclusion}

Cancer classification based on gene expression data is a trending research field in the area of data mining. The proposed algorithm in this study tended to the problem of early cancer detection which is important for proper cancer management. This paper presented a new methodology for the classification of human cancer based on their gene expression pattern. The proposed method deployed MI for feature selection while FA served as a 
feature selection framework; finally, SVM was used for the classification task. The performance of the method was evaluated on a colon microarray dataset and compared with other recent methods. From the results, FA was proved to be robust in achieving great results as it can predict using a lower number of genes compared to the other methods. In conclusion, the results of this study showed that the proposed method can maximize the accuracy of caner classification and minimize the number of selected genes compared to the other methods.

\section{References}

[1] S. Rawan and A. Manal, "Real time data analysis and visualization for the breast cancer disease," Period. Eng. Nat. Sci., vol. 7, no. 1, pp. 395-407, 2019.

[2] D. Y. Mahmood, A. G. Ismaeel, and A. H. Taqi, "Mining Method for Cancer and Pre-Cancer Detection Caused by Mutant Codon 248 in TP53," Period. Eng. Nat. Sci., vol. 7, no. 2, pp. 522-533, 2019.

[3] H. M. Alshamlan, G. H. Badr, and Y. A. Alohali, "Genetic bee colony (GBC) algorithm: a new gene selection method for microarray cancer classification," Comput. Biol. Chem., 2015.

[4] H. M. Alshamlan, "Co-ABC: Correlation artificial bee colony algorithm for biomarker gene discovery using gene expression profile," Saudi J. Biol. Sci., 2018.

[5] B. A. Logsdon and J. Mezey, "Gene expression network reconstruction by convex feature selection when incorporating genetic perturbations," PLoS Comput. Biol., 2010.

[6] A. M. Taha, S.-D. Chen, and A. Mustapha, "Natural Extensions: Bat Algorithm with Memory.," J. Theor. Appl. Inf. Technol., vol. 79, no. 1, pp. 1-9, 2015.

[7] A. M. Taha, S.-D. Chen, and A. Mustapha, "Bat Algorithm Based Hybrid Filter-Wrapper Approach," Adv. Oper. Res., vol. 2015, 2015.

[8] P. Moradi and M. Rostami, "Integration of graph clustering with ant colony optimization for feature selection," Knowledge-Based Syst., vol. 84, pp. 144-161, 2015.

[9] A. Al-ani, "Ant Colony Optimization for Feature Subset Selection,” vol. 1, no. 4, pp. 999-1002, 2007.

[10] S. M. Vieira, J. M. C. Sousa, and T. A. Runkler, "Two cooperative ant colonies for feature selection using fuzzy models," Expert Syst. Appl., vol. 37, no. 4, pp. 2714-2723, 2010.

[11] C.-H. Cheng, T.-L. Chen, and L.-Y. Wei, "A hybrid model based on rough sets theory and genetic algorithms for stock price forecasting," Inf. Sci. (Ny)., vol. 180, no. 9, pp. 1610-1629, 2010.

[12] M. M. Kabir, M. Shahjahan, and K. Murase, "A new local search based hybrid genetic algorithm for feature selection," Neurocomputing, vol. 74, no. 17, pp. 2914-2928, 2011.

[13] C.-H. Lin, H.-Y. Chen, and Y.-S. Wu, "Study of image retrieval and classification based on adaptive features using genetic algorithm feature selection," Expert Syst. Appl., vol. 41, no. 15, pp. 6611-6621, 2014.

[14] M. Schiezaro and H. Pedrini, "Data feature selection based on Artificial Bee Colony algorithm," $J$. Image Video Process., vol. 1, no. 47, pp. 1-8, 2013.

[15] V. Agrawal and S. Chandra, "Feature Selection using Artificial Bee Colony Algorithm for Medical Image Classification," 2015 Eighth Int. Conf. Contemp. Comput., vol. 1, pp. 2-7, 2015.

[16] B. Xue, M. Zhang, and W. N. Browne, "Particle swarm optimisation for feature selection in classification: Novel initialisation and updating mechanisms," Appl. Soft Comput. J., vol. 18, pp. 261276, 2014.

[17] B. Xue, M. Zhang, S. Member, and W. N. Browne, "Particle Swarm Optimization for Feature 
Selection in Classification : A Multi-Objective Approach,”pp. 1-16, 2012.

[18] C. C. O. Ramos, A. N. Souza, G. Chiachia, A. X. Falc??o, and J. P. Papa, "A novel algorithm for feature selection using Harmony Search and its application for non-technical losses detection," Comput. Electr. Eng., vol. 37, no. 6, pp. 886-894, 2011.

[19] H. H. Inbarani, M. Bagyamathi, and A. T. Azar, "A novel hybrid feature selection method based on rough set and improved harmony search," Neural Comput. Appl., vol. 26, no. 8, pp. 1859-1880, 2015.

[20] X. S. Yang, "Firefly algorithms for multimodal optimization," Lect. Notes Comput. Sci. (including Subser. Lect. Notes Artif. Intell. Lect. Notes Bioinformatics), vol. 5792 LNCS, pp. 169-178, 2009.

[21] X. she Yang, "Firefly algorithm, Levy flights and global optimization," Res. Dev. Intell. Syst., pp. 135$146,2010$.

[22] X. S. Yang and X. He, "Firefly algorithm: recent advances and applications," Int. J. Swarm Intell., vol. 1 , no. 1 , p. 36, 2013.

[23] H. A. Ahmed, M. F. Zolkipli, and M. Ahmad, "A novel efficient substitution-box design based on firefly algorithm and discrete chaotic map," Neural Computing and Applications, 2018.

[24] Z. M. Yaseen, I. Ebtehaj, H. Bonakdari, R. C. Deo, A. Danandeh Mehr, W. H. M. W. Mohtar, L. Diop, A. El-shafie, and V. P. Singh, "Novel approach for streamflow forecasting using a hybrid ANFIS-FFA model," J. Hydrol., vol. 554, pp. 263-276, 2017.

[25] M. A. Ghorbani, R. C. Deo, V. Karimi, Z. M. Yaseen, and O. Terzi, "Implementation of a hybrid MLP-FFA model for water level prediction of Lake Egirdir, Turkey," Stochastic Environmental Research and Risk Assessment, pp. 1-15, 2017.

[26] E. Bonilla Huerta, B. Duval, and J. K. Hao, "A hybrid LDA and genetic algorithm for gene selection and classification of microarray data," Neurocomputing, 2010.

[27] E. Alba, J. García-Nieto, L. Jourdan, and E. G. Talbi, "Gene selection in cancer classification using $\mathrm{PSO} / \mathrm{SVM}$ and GA/SVM hybrid algorithms," in 2007 IEEE Congress on Evolutionary Computation, CEC 2007, 2007.

[28] T. TUNCER, "SCSO: A novel sine-cosine based swarm optimization algorithm for numerical function optimization," Period. Eng. Nat. Sci., vol. 6, no. 2, pp. 1-9, 2018.

[29] S. Q. Salih and A. A. Alsewari, "Solving large-scale problems using multi-swarm particle swarm approach," Int. J. Eng. Technol., vol. 7, no. 3, pp. 1725-1729, 2018.

[30] S. Q. Salih, A. A. Alsewari, and Z. M. Yaseen, "Pressure Vessel Design Simulation: Implementing of Multi-Swarm Particle Swarm Optimization,” Proc. 2019 8th Int. Conf. Softw. Comput. Appl., pp. 120$124,2019$.

[31] W. Jing, Z. M. Yaseen, S. Shahid, M. K. Saggi, H. Tao, O. Kisi, S. Q. Salih, N. Al-Ansari, and K.-W. Chau, "Implementation of evolutionary computing models for reference evapotranspiration modeling: short review, assessment and possible future research directions," Eng. Appl. Comput. Fluid Mech., vol. 13, no. 1, pp. 811-823, 2019.

[32] Z. YILMAZ, M. OKSAR, and F. BASCIFTCI, "Multi-Objective Artificial Bee Colony Algorithm to Estimate Transformer Equivalent Circuit Parameters," Period. Eng. Nat. Sci., vol. 5, no. 3, pp. 271$277,2017$.

[33] S. Q. Salih, "A New Training Method Based on Black Hole Algorithm for Convolutional Neural Network," J. Sourthwest Jiaotong Univ., vol. 54, no. 3, pp. 1-10, 2019.

[34] S. F. Jabbar, R. I. Hamed, and A. H. Alwan, "The potential of nonparametric model in foundation bearing capacity prediction," Neural Comput. Appl., 2018. 
[35] A. A. Al-Musawi, A. A. H. Alwanas, S. Q. Salih, H. Z. Ali, M. T. Tran, and Z. M. Yaseen, "Shear strength of SFRCB without stirrups simulation: implementation of hybrid artificial intelligence model," Eng. Comput., pp. 1-11, 2019.

[36] Z. A. Al Sudani, S. Q. Salih, Z. M. Yaseen, and others, "Development of Multivariate Adaptive Regression Spline Integrated with Differential Evolution Model for Streamflow Simulation," J. Hydrol., pp. 1-15, 2019.

[37] A. Abdulelah, H. Alwanas, A. A. Al-musawi, S. Q. Salih, H. Tao, M. Ali, and Z. M. Yaseen, "Loadcarrying capacity and mode failure simulation of beam-column joint connection : Application of selftuning machine learning model," Eng. Struct., vol. 194, pp. 220-229, 2019.

[38] S. Ekiz, "Solving Constrained Optimization Problems with Sine-Cosine Algorithm," Period. Eng. Nat. Sci., vol. 5, no. 3, pp. 378-386, 2017.

[39] A. Jovic, K. Brkic, and N. Bogunovic, "A review of feature selection methods with applications," Ieee, pp. 1200-1205, 2015.

[40] J. Tang, S. Alelyani, and H. Liu, "Feature Selection for Classification: A Review," Data Classif. Algorithms Appl., pp. 37-64, 2014.

[41] M. Bayati and S. Jabbar, "Developing a Spam Email Detector," Int J Eng Innov Technol, vol. 5, no. 2, pp. 16-21, 2015.

[42] R. Vega, T. Sajed, K. W. Mathewson, K. Khare, P. M. Pilarski, R. Greiner, G. Sanchez-Ante, and J. M. Antelis, "Assessment of feature selection and classification methods for recognizing motor imagery tasks from electroencephalographic signals," Artif. Intell. Res., vol. 6, no. 1, p. 37, 2016.

[43] A. F. Cabrera, D. Farina, and K. Dremstrup, "Comparison of feature selection and classification methods for a brain--computer interface driven by non-motor imagery," Med. Biol. Eng. Comput., vol. 48, no. 2, pp. 123-132, 2010.

[44] T. Alotaiby, F. E. A. El-Samie, S. A. Alshebeili, and I. Ahmad, "A review of channel selection algorithms for EEG signal processing,” EURASIP J. Adv. Signal Process., 2015.

[45] E. Emary, H. M. Zawbaa, K. K. A. Ghany, A. E. Hassanien, and B. Parv, "Firefly Optimization Algorithm for Feature Selection," Proc. 7th Balk. Conf. Informatics Conf. - BCI '15, pp. 1-7, 2015.

[46] A. Kulkarni, B. S. C. Naveen Kumar, V. Ravi, and U. S. Murthy, "Colon cancer prediction with genetics profiles using evolutionary techniques," Expert Syst. Appl., 2011.

[47] M. S. Mohamad, S. Deris, and R. M. Illias, "A hybrid of genetic algorithm and support vector machine for features selection and classification of gene expression microarray," Int. J. Comput. Intell. Appl., vol. 5, no. 01, pp. 91-107, 2005.

[48] S. Cho and H. Won, "Machine Learning in DNA Microarray Analysis for Cancer Classification," in Proceedings of the First Asia-Pacific bioinformatics conference on Bioinformatics 2003, 2003.

[49] J.-Y. Yeh, "Applying data mining techniques for cancer classification on gene expression data," Cybern. Syst. An Int. J., vol. 39, no. 6, pp. 583-602, 2008.

[50] O. H. Fang, N. Mustapha, and M. N. Sulaiman, "Integrating biological information for feature selection in microarray data classification," in 2010 Second International Conference on Computer Engineering and Applications, 2010, pp. 330-334.

[51] S. M. Alladi, P. Shinde Santosh, V. Ravi, and U. S. Murthy, "Colon cancer prediction with genetic profiles using intelligent techniques," Bioinformation, vol. 3, no. 3, p. 130, 2008. 